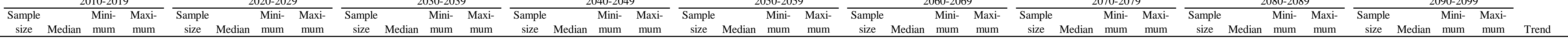

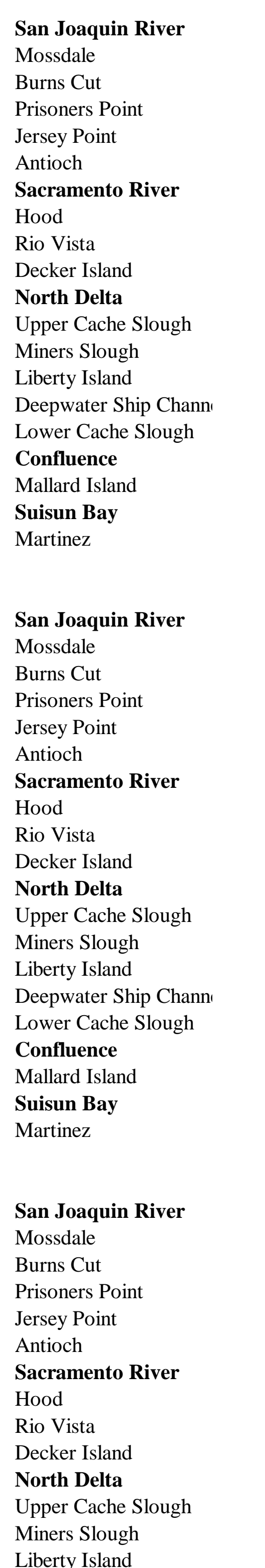

\begin{tabular}{|c|c|c|c|c|c|c|}
\hline 775.0 & 145 & 195 & 10 & 171.5 & 147 & 19 \\
\hline 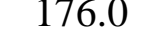 & 160 & 190 & 10 & 170.0 & 166 & \\
\hline 88.0 & 174 & 223 & 10 & 185.5 & 16 & \\
\hline 193.0 & 176 & 259 & 10 & 195.5 & 18 & \\
\hline 194.0 & 173 & 232 & & 189.0 & 16 & \\
\hline 237.0 & 198 & 260 & 10 & 222.0 & 19 & \\
\hline & 185 & 237 & 10 & 209.0 & 18 & \\
\hline 216.0 & 187 & 263 & 10 & 209.5 & 18 & \\
\hline 239.0 & 201 & & 8 & & 19 & \\
\hline 240.0 & & 266 & 9 & 230.0 & 19 & \\
\hline 215.0 & 178 & 263 & 10 & 213.5 & & \\
\hline 225.5 & 200 & 264 & 10 & 218.5 & 19 & \\
\hline & & & & 224.0 & & \\
\hline 231.5 & 195 & 261 & 10 & 221.5 & 19 & \\
\hline 70 & $22:$ & 267 & 4 & 253.0 & 0 & 28 \\
\hline
\end{tabular}

Scenario GFDL-A2

$\begin{array}{llllllllllllllllllll}195.0 & 169 & 206 & 10 & 178.5 & 166 & 221 & 10 & 166.5 & 127 & 217 & 10 & 164.0 & 155 & 182 & 10 & 165.5 & 143 & 187 \\ 188.0 & 165 & 197 & 10 & 180.0 & 170 & 202 & 10 & 169.5 & 149 & 205 & 10 & 164.5 & 154 & 179 & 10 & 168.5 & 146 & 184\end{array}$

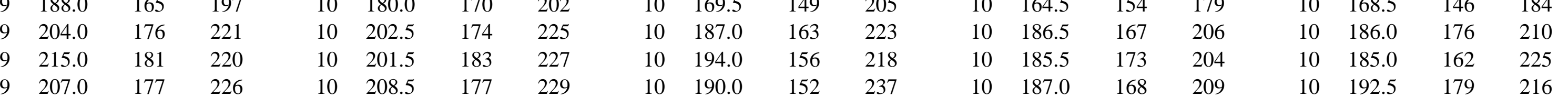

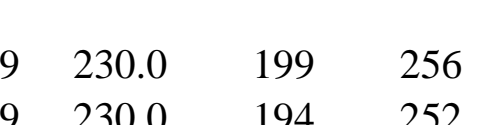
$\begin{array}{llll}10 & 235.5 & 206 & 256\end{array}$

$\begin{array}{llll} & 230.0 & 194 & 252 \\ 9 & 225.0 & 195 & 259\end{array}$

$\begin{array}{rrrrr}9 & 208.0 & 176 & 228 \\ 10 & 205.5 & 174 & 243 \\ 9 & 204.0 & 167 & 222\end{array}$

$\begin{array}{llll}9 & 251.0 & 209 & 279 \\ 9 & 250.0 & 210 & 277 \\ 9 & 228.0 & 198 & 262 \\ 9 & 237.0 & 207 & 273\end{array}$

$\begin{array}{llll}9 & 2237.0 & 207 & 273 \\ 9 & 237.0 & 208 & 277\end{array}$

$\begin{array}{llll}9 & 233.0 & 204 & 265\end{array}$

$\begin{array}{llll}7 & 245.0 & 193 & 275 \\ 9 & 244.0 & 212 & 277\end{array}$

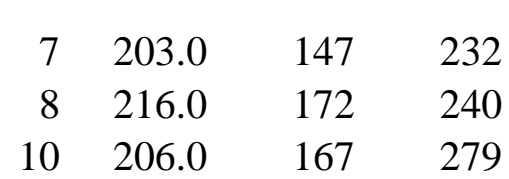

$\begin{array}{llll}10 & 2105.0 & 185 & 234 \\ 10 & 206.5 & 184 & 227 \\ & & & \end{array}$

$\begin{array}{llll}10 & 215.0 & 198 & 244\end{array}$

$\begin{array}{llll}10 & 214.0 & 192 & 234 \\ 10 & 207.0 & 174 & 232\end{array}$

$\begin{array}{llll}242.0 & 193 & 272 \\ 2440 & 210 & 272\end{array}$

$\begin{array}{rrrr}8 & 202.5 & 147 & 235 \\ 8 & 205.0 & 147 & 236\end{array}$

$\begin{array}{llll}10 & 204.5 & 185 & 248 \\ 10 & 219.0 & 186 & 246 \\ 10 & 193.5 & 179 & 240 \\ 1 & 19.5 & 18 & 244\end{array}$

$\begin{array}{llll}8 & 225.0 & 182 & 247 \\ 9 & 233.0 & 183 & 245 \\ 1 & 207.0 & 167 & 237\end{array}$

$\begin{array}{llll}10 & 207.0 & 167 & 237 \\ 10 & 208.0 & 180 & 238 \\ 10 & 217.5 & 180 & 245\end{array}$

$\begin{array}{llll}2 & 244.0 & 232 & 256\end{array}$

$\begin{array}{llll}5 & 248.0 & 210 & 215\end{array}$

$\begin{array}{rrr}206.5 & 175 & 244 \\ 38.5 & 204 & 243\end{array}$

$\begin{array}{llll}10 & 203.5 & 185 & 233\end{array}$

$\begin{array}{llll}10 & 215.5 & 198 & 253\end{array}$

$\begin{array}{lll}240.0 & 203 \quad 272\end{array}$

\title{
Scenario PCM-A2
}

$\begin{array}{llllllllllllllllllll}9 & 183.0 & 166 & 221 & 10 & 182.5 & 166 & 204 & 10 & 175.0 & 158 & 181 & 10 & 177.5 & 142 & 196 & 10 & 159.0 & 133 & 168 \\ 9 & 180.0 & 176 & 211 & 10 & 176.5 & 162 & 193 & 10 & 174.0 & 157 & 189 & 10 & 171.0 & 142 & 194 & 10 & 162.0 & 146 & 180 \\ 9 & 223.0 & 169 & 234 & 10 & 194.0 & 181 & 210 & 10 & 198.0 & 185 & 218 & 10 & 191.5 & 176 & 218 & 10 & 187.0 & 157 & 200 \\ 9 & 221.0 & 188 & 259 & 10 & 20.0 & 182 & 211 & 10 & 197.0 & 181 & 222 & 10 & 191.0 & 165 & 221 & 10 & 187.5 & 149 & 220\end{array}$

$10 \quad 224.5 \quad 193 \quad 255$

$\begin{array}{llll}10 & 207.5 & 169 & 230\end{array}$

$\begin{array}{lll}253.0 & 212 & 276 \\ 238.0 & 201 & 259 \\ 238.5 & 199 & 280\end{array}$

$\begin{array}{rrrr}9 & 214.0 & 196 & 245 \\ 10 & 213.0 & 191 & 239 \\ 10 & 2120 & 196 & 235\end{array}$

$\begin{array}{llll}10 & 223.0 & 207 & 240 \\ 10 & 211.5 & 195 & 235 \\ 10 & 218.0 & 195 & 226\end{array}$

$\begin{array}{lll}214.5 & 185 & 249 \\ 198.0 & 174 & 248\end{array}$

$\begin{array}{llll}10 & 202.5 & 166 & 219 \\ 10 & 203.0 & 153 & 227\end{array}$

$\begin{array}{llll}10 & 160.5 & 143 & 180 \\ 10 & 159.0 & 128 & 177 \\ 10 & 170.5 & 155 & 184\end{array}$

$\begin{array}{llll}10 & 149.5 & 140 & 180 \\ 10 & 148.0 & 142 & 169 \\ 10 & 1595 & 147 & 194\end{array}$

$\begin{array}{llll}10 & 133.5 & 110 & 160 \\ 141.5 & 119 & 169\end{array}$

$\begin{array}{lllll}10 & 136.0 & 120 & 145 & -5.77 * * \\ 10 & 136.5 & 122 & 161 & -5.20 * * \\ 10 & 1625 & 138 & 167 & -59 * * *\end{array}$

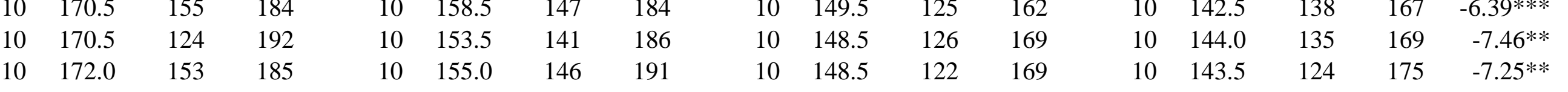

$\begin{array}{llllllll}10 & 197.0 & 168 & 224 & 10 & 183.5 & 152 & 222 \\ 10 & 188.5 & 160 & 206 & 10 & 1770 & 150 & 204\end{array}$

$\begin{array}{llll}10 & 169.5 & 158 & 196 \\ 10 & 161.5 & 152 & 195\end{array}$

$\begin{array}{llllll}10 & 160.0 & 145 & 186 & -9.59 * * * * \\ 10 & 157.5 & 140 & 179 & -8.5 \% * * * \\ 10 & 155.0 & 139 & 171 & -8.54 * * *\end{array}$

$\begin{array}{llll}10 & 199.0 & 167 & 240 \\ 10 & 1960 & 169 & 236\end{array}$

$\begin{array}{llll}10 & 177.0 & 150 & 204 \\ 10 & 162.5 & 154 & 189\end{array}$

$\begin{array}{llll}10 & 162.5 & 152 & 195 \\ 128 & 182\end{array}$

$\begin{array}{llll}10 & 175.0 & 153 & 200 \\ 10 & 181.0 & 156 & 209 \\ 10 & 163.0 & 146 & 191 \\ 10 & 172.5 & 151 & 198 \\ 10 & 1735 & 151 & 200\end{array}$

$\begin{array}{llll}0 & 165.5 & 132 & 193 \\ 0 & 171.0 & 133 & 193 \\ 0 & 159.5 & 131 & 188 \\ 0 & 153.0 & 128 & 179\end{array}$

$\begin{array}{llllll}10 & 161.5 & 134 & 180 & -10.8^{* * *} \\ 10 & 163.0 & 142 & 180 & -10.29^{* * *} \\ 1 & 15.5 & 127 & 179 & -9.44 *\end{array}$ $\begin{array}{llll}10 & 188.5 & 165 & 21 \\ 10 & 194.0 & 166 & 235\end{array}$

$\begin{array}{llll}10 & 189.5 & 160 & 217\end{array}$

$\begin{array}{llll}10 & 180.5 & 150 & 22\end{array}$

$\begin{array}{llll}10 & 165.5 & 147 & 200\end{array}$

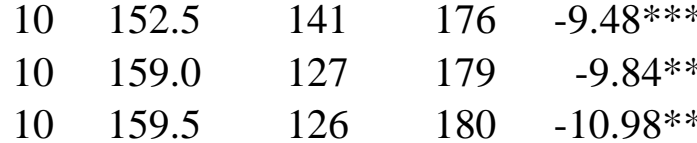

$10 \begin{array}{lllll}107.5 & 142 & 180 & -10.01 * * *\end{array}$

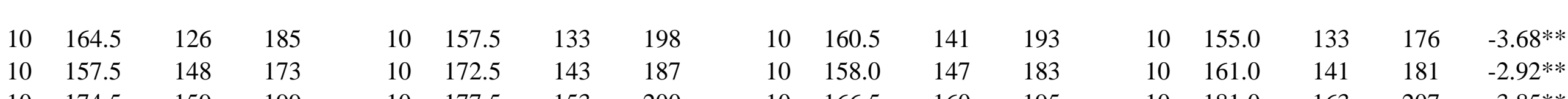

$$
\begin{array}{llll}
10 & 190.0 & 175 & 226
\end{array}
$$

$\begin{array}{lll}174.0 & 159 & 203\end{array}$

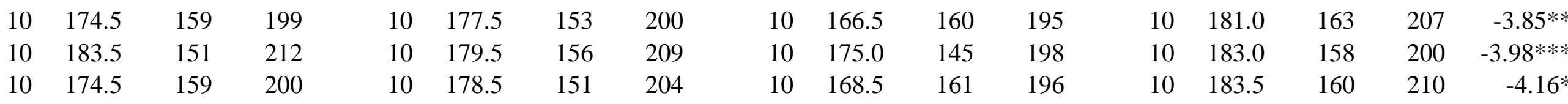
$\begin{array}{lllllllllllllllll}10 & 203.5 & 185 & 223 & 10 & 196.0 & 161 & 232 & 10 & 201.5 & 174 & 215 & 10 & 201.5 & 184 & 217 & -4.11^{*} \\ 10 & 194.0 & 183 & 208 & 10 & 188.0 & 160 & 208 & 10 & 187.5 & 169 & 204 & 10 & 198.5 & 180 & 216 & -4.93^{* *} \\ 10 & 191.5 & 173 & 224 & 10 & 188.5 & 164 & 214 & 10 & 189.0 & 164 & 205 & 10 & 194.5 & 179 & 209 & -4.58^{*}\end{array}$ $\begin{array}{llllllll}9 & 200.0 & 174 & 235 & 10 & 208.0 & 162 & 27 \\ 9 & 202.0 & 175 & 232 & 10 & 205.5 & 162 & 230 \\ 10 & 1690 & 232 & 10 & 1655 & 157 & 216\end{array}$

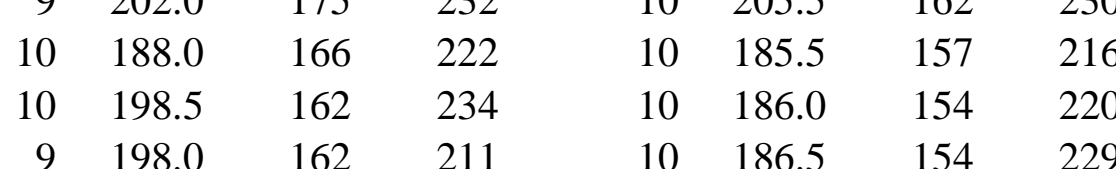
$10 \quad 192.0 \quad 185 \quad 224$ $\begin{array}{llll}10 & 186.5 & 154 & 229 \\ 10 & 190.0 & 160 & 232\end{array}$ $\begin{array}{llll}8 & 197.0 & 176 & 235\end{array}$ $\begin{array}{llll}10 & 202.0 & 151 & 232 \\ 10 & 202.5 & 167 & 231 \\ 10 & 187.5 & 147 & 207 \\ 10 & 192.5 & 15 & 228\end{array}$ $\begin{array}{llll}10 & 19.5 & 151 & 228 \\ 10 & 193.0 & 151 & 230\end{array}$

$\begin{array}{llll}10 & 200.0 & 166 & 236\end{array}$ $\begin{array}{llllr}9 & 197.0 & 156 & 209 & -5.66^{*} \\ 9 & 199.0 & 185 & 212 & -6.11^{* *}\end{array}$

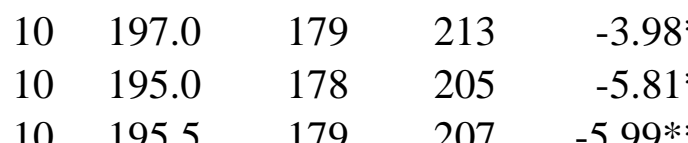
$\begin{array}{lllll}10 & 200.5 & 182 & 217 & -5.76^{*}\end{array}$ $\begin{array}{rrrrrrrrrrrrrrrrrrrr}3 & 250.0 & 229 & 276 & 9 & 229.0 & 191 & 292 & 7 & 235.0 & 206 & 255 & 10 & 238.0 & 193 & 266 & 10 & 219.5 & 158 & 265 \\ 4 & 262.5 & 231 & 277 & 10 & 235.5 & 191 & 292 & 8 & 234.0 & 206 & 247 & 10 & 233.5 & 192 & 265 & 10 & 219.5 & 159 & 247 \\ 9 & 238.0 & 198 & 279 & 10 & 214.5 & 184 & 223 & 10 & 215.0 & 189 & 228 & 10 & 197.0 & 169 & 255 & 10 & 201.5 & 153 & 239\end{array}$

\begin{tabular}{llll}
10 & 161.5 & 142 & 184 \\
\hline & 165 & 142 & 192
\end{tabular} $\begin{array}{llll}10 & 165.0 & 149 & 18 \\ 10 & 163.0 & 148 & 177 \\ 10 & 180.0 & 156 & 198 \\ 10 & 177.0 & 157 & 203 \\ 10 & 182.0 & 155 & 198\end{array}$ $\begin{array}{llllllll}10 & 206.5 & 173 & 244 & 10 & 205.0 & 182 & 237 \\ 10 & 192.5 & 166 & 219 & 10 & 194.0 & 162 & 210 \\ 10 & 187.0 & 168 & 210 & 10 & 185.0 & 162 & 226\end{array}$ $\begin{array}{llll}9 & 215.0 & 171 & 231 \\ 9 & 2180 & 187 & 223\end{array}$ $\begin{array}{llll}10 & 201.5 & 178 & 238 \\ 10 & 206.0 & 178 & 237 \\ 10 & 184.0 & 163 & 2\end{array}$ $\begin{array}{lllllllll}10 & 155.0 & 120 & 167 & 10 & 147.0 & 119 & 179 & -4.38 * * \\ 10 & 153.5 & 131 & 169 & 10 & 147.5 & 140 & 176 & -3.86 * * * \\ 10 & 17.5 & 138 & 162 & & 10 & & \end{array}$ $\begin{array}{lllllllll}10 & 164.5 & 140 & 190 & 10 & 159.5 & 149 & 184 & -6.44^{* * * *} \\ 10 & 172.5 & 138 & 182 & 10 & 161.5 & 148 & 186 & -6.21+* * * \\ 10 & 164.0 & 135 & 191 & 10 & 155.0 & 149 & 187 & -7.35^{* * *}\end{array}$ $\begin{array}{lllllllll}10 & 200.5 & 172 & 209 & 10 & 192.5 & 167 & 205 & -5.61^{* * *} \\ 10 & 185.0 & 166 & 200 & 10 & 180.0 & 163 & 202 & -6.22^{* * *} \\ 10 & 181.5 & 148 & 190 & 10 & 172.5 & 154 & 190 & -7.22^{* * *}\end{array}$ $\begin{array}{lllllllll}10 & 209.5 & 169 & 230 & 10 & 190.0 & 162 & 223 & -6.48^{* * *} \\ 10 & 209.0 & 169 & 228 & 10 & 195.0 & 165 & 219 & -7.02^{* * *} \\ 10 & & & & & & & & \end{array}$

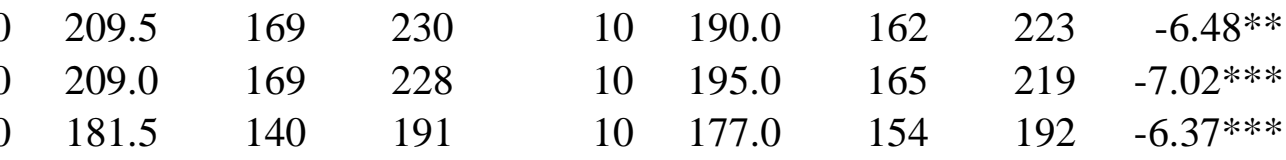




\begin{tabular}{|c|c|c|c|c|c|c|c|c|c|c|c|c|c|c|c|c|c|c|c|c|c|c|c|c|c|c|c|c|c|c|c|c|c|c|c|c|c|}
\hline wwater Ship Channı & & & 228 & 273 & 10 & & 184 & 289 & & 227.5 & & & 10 & 230.5 & 190 & 254 & 10 & 197.5 & 156 & 237 & 102 & 201.5 & 165 & & 10 & 188.5 & & 229 & 10 & 194.5 & 162 & 217 & 10 & 176.0 & 161 & 198 & \\
\hline $\begin{array}{l}\text { Lower Cache Slough } \\
\text { Confluence }\end{array}$ & & 258.5 & 229 & 276 & 10 & 225.5 & 184 & 291 & 92 & 230.0 & 203 & 237 & 10 & 232.5 & 191 & 265 & 10 & 205.5 & 157 & 239 & 102 & 212.5 & 166 & 258 & 10 & 192.5 & 175 & 236 & 10 & 205.5 & 163 & 227 & 10 & 188.5 & 160 & 199 & \\
\hline $\begin{array}{l}\text { Mallard Island } \\
\text { Suisun Bay }\end{array}$ & 6 & 249.0 & 215 & 282 & 9 & 232.0 & 202 & 275 & 102 & 226.5 & 207 & 241 & 10 & 233.5 & 191 & 256 & 10 & 211.5 & 168 & 227 & 92 & 209.0 & 172 & 223 & 10 & 201.5 & 182 & 238 & 10 & 202.0 & 147 & 226 & 10 & 192.0 & 165 & 216 & \\
\hline Martinez & 1 & 237.0 & 237 & 237 & 2 & 242.0 & 239 & 245 & $0 \mathrm{NV}$ & & $\mathrm{NV} \quad \mathrm{N} /$ & JV & 4 & 217.0 & 196 & 265 & 7 & 236.0 & 217 & 256 & 82 & 221.5 & 195 & 232 & 9 & 231.0 & 191 & 258 & 10 & 222.0 & 178 & 238 & 10 & 211.5 & 172 & 228 & \\
\hline & \multirow{2}{*}{\multicolumn{37}{|c|}{ Scenario PCM-B1 }} \\
\hline $\begin{array}{l}\text { Joaquin River } \\
\text { sdale }\end{array}$ & & & & & & & & & & & & & & & & & & & & & & & & & & & & & & & & & & & & & \\
\hline Burns $\mathrm{C}$ & & $\begin{array}{l}18.0 \\
177.0\end{array}$ & 147 & $\begin{array}{l}197 \\
192\end{array}$ & 10 & $\begin{array}{l}18.9 .5 \\
177.0\end{array}$ & 159 & $\begin{array}{l}194 \\
190\end{array}$ & 10 & $\begin{array}{l}100.5 \\
175.5\end{array}$ & $\begin{array}{l}155 \\
155\end{array}$ & $\begin{array}{l}150 \\
191\end{array}$ & 10 & $\begin{array}{l}183.0 \\
177.0\end{array}$ & $\begin{array}{l}156 \\
155\end{array}$ & 202 & 10 & $\begin{array}{l}1 / 76.0 \\
174.0\end{array}$ & 149 & $\begin{array}{l}188 \\
181\end{array}$ & 10 & $\begin{array}{l}16.5 \\
169.5\end{array}$ & 165 & 204 & 10 & $\begin{array}{l}10530.0 \\
1630\end{array}$ & 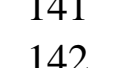 & 182 & 10 & $\begin{array}{l}169.0 \\
157.0\end{array}$ & 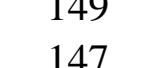 & 1948 & 10 & $\begin{array}{l}11 / .5 \\
168.0\end{array}$ & 145 & 185 & \\
\hline $\begin{array}{l}\text { Prisoners } \\
\text { Plo }\end{array}$ & 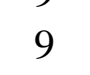 & 202.0 & 185 & & 10 & 208.5 & 188 & 243 & 10 & 194.0 & 173 & 213 & 10 & 196.0 & 184 & 208 & 10 & $\begin{array}{l}1 / 4.0 \\
185.5\end{array}$ & 176 & 225 & 10 & 194.5 & 177 & 219 & 0 & 186.0 & 156 & 203 & 0 & 181.0 & 160 & 08 & 10 & $\begin{array}{l}0.0 .5 \\
85.5\end{array}$ & 9 & 0 & \\
\hline th & 9 & 211.0 & 178 & & 10 & 206.5 & 182 & 226 & 10 & 1922.5 & 165 & 218 & 10 & & & & 10 & 189.5 & 179 & 233 & 10 & 197.0 & 165 & 223 & 0 & & 63 & & & & 53 & 15 & 0 & & 5 & 7 & \\
\hline $\begin{array}{l}\text { Antioch } \\
\text { nacramen }\end{array}$ & 9 & 203.0 & 188 & 255 & 10 & 212.0 & 194 & 245 & 10 & 188.0 & 168 & 228 & 10 & 198.0 & 185 & 215 & 10 & 186.0 & 162 & 230 & 10 & 198.5 & 178 & 222 & 10 & 180.0 & 155 & 200 & 10 & 183.5 & 157 & 211 & 10 & 185.5 & 159 & 203 & \\
\hline Hood & 8 & 245.0 & 231 & 268 & 10 & 241.0 & 211 & 258 & 10 & 243.5 & 189 & 258 & 10 & 232.5 & 192 & 276 & 10 & 216.5 & 185 & 245 & 10 & 218.5 & 184 & & 10 & & & 5 & 10 & 9.0 & 80 & 34 & 10 & 197.5 & 1 & & \\
\hline & $\begin{array}{l}9 \\
0\end{array}$ & 234.0 & & & 10 & 225.5 & & & 10 & 207.0 & & & 10 & & & & & & & & 10 & & & & 10 & & & & & & & & & & 57 & & \\
\hline $\begin{array}{l}\text { Decker Islatad } \\
\text { North Delta }\end{array}$ & 8 & 235.5 & 218 & 243 & 10 & 233.5 & 203 & 261 & 10 & 214.5 & 179 & 246 & 10 & 213.5 & 174 & 252 & 10 & 208.5 & 184 & 244 & 10 & 206.0 & 172 & 235 & 10 & 205.5 & 167 & 218 & 10 & 193.0 & 175 & 229 & 0 & 2000.0 & 168 & 18 & \\
\hline Slough & 3 & 264.0 & 236 & & 4 & 243.0 & & & 4 & & 189 & 26: & 5 & & & 25 & 10 & 233.5 & & 27. & 8 & & 170 & & 9 & & 167 & & 9 & 5.0 & 78 & & 10 & 220.0 & i9 & 5 & \\
\hline & & 249.0 & & & 8 & 249.5 & & & 8 & & & & 8 & & & & & & & & 10 & & & & 9 & & & & & & & & & & & & \\
\hline & 8 & $\begin{array}{l}2223.5 \\
2350\end{array}$ & & & $\begin{array}{c}10 \\
9\end{array}$ & $\begin{aligned} 2195 \\
2709\end{aligned}$ & & & $\begin{array}{c}10 \\
9\end{array}$ & & & & $\begin{array}{c}10 \\
9\end{array}$ & & & & $\begin{array}{l}10 \\
10\end{array}$ & & & & $\begin{array}{l}10 \\
10\end{array}$ & & & & $\begin{array}{l}10 \\
10\end{array}$ & & $\beta_{5}^{3}$ & & P & & 5 & & 然 & $\begin{array}{l}192.5 \\
9125\end{array}$ & 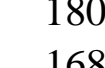 & 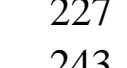 & \\
\hline 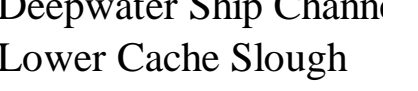 & 7 & 244.0 & 228 & 276 & 8 & 244.0 & 223 & 268 & 9 & 239.0 & 188 & 264 & 8 & $\begin{array}{l}21950.0 \\
235.5\end{array}$ & 177 & $\begin{array}{l}265 \\
268\end{array}$ & 10 & $\begin{array}{l}228.5 \\
230.5\end{array}$ & $\begin{array}{l}182 \\
182\end{array}$ & 262 & 10 & $\begin{array}{l}222.0 \\
223.5\end{array}$ & $\begin{array}{l}170 \\
170\end{array}$ & $\begin{array}{l}240 \\
261\end{array}$ & 10 & 208.5 & 165 & $\begin{array}{l}231 \\
237\end{array}$ & 10 & $\begin{array}{l}197.0 \\
208.0\end{array}$ & 176 & 236 & 10 & $\begin{array}{l}213.5 \\
218.5\end{array}$ & 168 & 245 & \\
\hline $\begin{array}{l}\text { flutur Is } \\
\text { lat }\end{array}$ & 7 & 249.0 & 234 & 262 & 8 & 244.0 & 222 & 268 & 9 & 247.0 & 193 & 266 & 8 & 230.5 & 191 & 281 & 10 & 233.0 & 185 & 244 & 10 & 228.5 & 184 & 245 & 10 & 203.0 & 168 & 237 & 10 & 209.0 & 172 & 237 & 10 & 204.5 & 170 & 242 & \\
\hline Suisun Bay & & & 255 & & 2 & 30 & 229 & 247 & & 0 & 221 & 221 & 1 & 44.0 & 244 & 244 & & 246.5 & 202 & 253 & 4 & 242.0 & 238 & 252 & & 435 & 204 & 266 & 6 & 2385 & 199 & 250 & & 2235 & 177 & 246 & \\
\hline
\end{tabular}

